\title{
Ventral tegmental area deep brain stimulation for chronic cluster headache: Effects on cognition, mood, pain report behaviour and quality of life
}

Davide Cappon ${ }^{1,5+}$,Agata Ryterska ${ }^{1,2+}$, Susie Lagrata ${ }^{3}$, Sarah Miller $^{3}$, Harith Akram ${ }^{1}$, Jonatham Hyam ${ }^{1}$, Ludvic Zrinzo ${ }^{1}$, Manjit Matharu ${ }^{3}$, Marjan Jahanshahi ${ }^{1,4 *}$.

${ }^{1}$ Unit of Functional Neurosurgery, Sobell Department of Motor Neuroscience \& Movement Disorders, UCL Institute of Neurology

2 Department of Psychology, Queen Mary, London University

${ }^{3}$ Headache Group, the National Hospital for Neurology and Neurosurgery

${ }^{4}$ The Clinical Hospital of Chengdu Brain Science Institute, MOE Key Lab for Neuroinformation, University of Electronic Science and Technology of China, Chengdu, China

${ }^{5}$ Berenson-Allen Center for Noninvasive Brain Stimulation, Division of Cognitive Neurology, Beth Israel Deaconess Medical Center, Harvard Medical School, Boston, MA, USA

+ These authors contributed equally to this work

* correspondence addressed to: Prof. M Jahanshahi, m.jahanshahi@ucl.ac.uk

Acknowledgments: AR was funded by $3+1 \mathrm{PhD}$ studentship, Queen Mary College, London University, HA was funded by the Brain Research Trust. The Unit of Functional Neurosurgery is supported by the Parkinson Appeal UK and the Monument Trust 


\begin{abstract}
Background

Deep brain stimulation in the ventral tegmental area (VTA-DBS) has provided remarkable therapeutic benefits in decreasing headache frequency and severity in patients with medically refractory chronic cluster headache $(\mathrm{CH})$. However, to date the effects of VTA-DBS on cognition, mood and quality of life have not been examined in detail.
\end{abstract}

\title{
Methods
}

The aim of the present study was to do so in a case series of 18 consecutive patients with $\mathrm{CH}$ who underwent implantation of DBS electrodes in the VTA. The patients were evaluated preoperatively and after a mean of 14 months of VTA-DBS on tests of global cognition (Mini Mental State Examination), intelligence (Wechsler Abbreviated Scale of Intelligence), verbal memory (California Verbal Learning Test-II), executive function (D-KEFS Stroop, verbal fluency, Trailmaking), and attention (Paced Serial Addition Test). Depression (Beck Depression Inventory (BDI), Hospital Anxiety Depression Scale (HADS)-Depression), anxiety (HADS-A), apathy (Starkstein Apathy Scale, SAS), hopelessness (Beck Hopelessness Scale, BHS), were also assessed. Subjective pain experience and behaviour (McGill Pain Questionnaire, Pain Behaviour Checklist) and quality of life (SF-36) were also evaluated at the same time points.

\section{Results}

VTA-DBS resulted in significant improvement of headache frequency (from mean of 5 to 2 episodes, $\mathrm{p}<.001$ ) and severity (from mean of 10 to $7, \mathrm{p}<.001$ ) which was associated with significant reduction of anxiety (from mean of 11.94 to $8.00, p<.001$ ) and help-seeking behaviours (from mean of 4.00 to $2.61, \mathrm{p}<.001$ ). VTA-DBS did not produce any significant change on any tests of cognitive function and any other outcome measures (BDI, HAD-D, SAS, BHS, McGill Pain Questionnaire, SF36). 


\section{Conclusion}

We confirm the efficacy of VTA-DBS in the treatment of medically refractory chronic $\mathrm{CH}$. The reduction of headache frequency and severity was associated with a significant reduction of anxiety. Furthermore, the result suggests that VTA-DBS for chronic $\mathrm{CH}$ improves pain-related help-seeking behaviours and does not produce any change in cognition.

\section{Key Words}

Trigeminal autonomic cephalalgia, VTA, DBS, cognition, anxiety. 


\section{Introduction}

Cluster headache $(\mathrm{CH})$ is a rare trigeminal autonomic cephalalgia (TAC) characterized by attacks of severe, cranial pain associated with ipsilateral autonomic symptoms such as conjunctival injection, lacrimation, nasal congestion, ptosis or eyelid oedema ${ }^{1} . \mathrm{CH}$ is one of the most excruciating and disabling conditions and it has a severe impact on the patient's quality of life. Patients with $\mathrm{CH}$ show greater anxiety, depression and worse quality of life compared with healthy populations ${ }^{2}$. The exact pathophysiology in cluster headache $(\mathrm{CH})$ is matter of debate ${ }^{3-10}$. The seasonality and periodicity of $\mathrm{CH}$ attacks are indicative of a possible hypothalamic role ${ }^{7,11}$. This has been sustained by neuro-endocrinological studies ${ }^{12,13}$ and neuroimaging studies ${ }^{14,15}$. Early imaging studies pointed to increased activity and neuronal density in what was thought to be 'the posterior hypothalamic region' ${ }^{16}$. Further studies have shown this region to lie not in the hypothalamus but in the ventral tegmental area (VTA) ${ }^{17-19}$. In the course of an attack, pathological activation of the trigeminoparasympathetic brainstem reflex is considered to cause trigeminal nerve and craniofacial parasympathetic activation resulting in distinctive ipsilateral cranial pain and autonomic features 7,20. The trigemino-hypothalamic tract, which connects the posterior hypothalamic region to the trigeminal nucleus in the brainstem has been proposed as an important pathway in this process ${ }^{21}$. Standard medical treatments of $\mathrm{CH}$ include acute therapy aimed at aborting individual attacks, and preventative medications, which reduce the frequency of attacks. These treatments are effective for a proportion of the patients ${ }^{22}$. On the other hand, in a small but significant number of highly disabled individuals, standard medical therapy is not sufficiently effective and $\mathrm{CH}$ attacks are intractable. For these patients, deep brain stimulation (DBS) is considered as a therapeutic option. DBS is a surgical treatment in which electric pulses are continuously applied via stereotactically implanted electrodes. The first DBS procedure for $\mathrm{CH}$ in $2001^{23}$ targeted the so called "posteromedial hypothalamus" with positive results. Subsequent investigators ${ }^{18,19,21}$ have highlighted that the region being targeted is more accurately termed the VTA and have also reported beneficial effects of DBS of the VTA with significant reduction in the severity and the frequency of $\mathrm{CH}$ 
attacks ${ }^{17}$. The aim of the present study was to investigate the effect of VTA-DBS for $\mathrm{CH}$ on cognition, mood, pain experience and behaviour, and quality of life in most of the patients who were also included in the clinical report of Akram et al (2016).

\section{Methods}

\section{Study population}

Eighteen consecutive patients (3 female) with chronic $\mathrm{CH}(\mathrm{CCH})$, referred for VTA-DBS at the National Hospital for Neurology and Neurosurgery were enrolled. The patients met the International Classification of Headache Disorders-II diagnostic criteria for $\mathrm{CCH}^{1}$ and had experienced highly disabling, medically refractory symptoms for at least 24 months. $\mathrm{CCH}$ was considered medical refractory if patients failed to respond to at least 5 of the following 7 drugs: verapamil, lithium, methysergide, topiramate, melatonin, gabapentin and valproate. Failure was defined as one of the following: unsatisfactory response, side effects intolerance or contraindication to the use. Moreover, all patients were considered for occipital nerve stimulation (ONS) prior to DBS and had either been refused funding or did not have a good response. This study was approved by the Local Ethics Committee. All participants gave written informed consent. Demographic and clinical details of the patients are presented in Table 1.

\section{Procedure}

All enrolled participants underwent clinical examination and neuropsychological assessment prior to surgery. Clinical and neuropsychological assessments were repeated $14 \pm 4.5$ months postoperatively. The surgical procedure has been described previously ${ }^{24}$ and involved DBS lead (model 3389, Medtronic Inc.) implantation in the ipsilateral VTA or bilaterally (if symptoms were side alternating) under local or general anesthesia. The most deep contact location of the quadripolar lead was determined on a 1.5T T2 axial stereotactic MRI at a level directly above the mammillary bodies, anteromedial to the hypointense red nucleus and posterolateral to the 
hypointense mammillothalamic tract ${ }^{24}$. In the weeks following surgery, open label programming was conducted to define optimal stimulation parameters. All devices were programmed with a frequency of $185 \mathrm{~Hz}$ and pulse width of $60 \mu \mathrm{s}$, gradually increasing the intensity up to 4.0 volts (V) on weekly intervals according to occurrence of side effects (diplopia, vertigo, oscillopsia, and ophthalmoplegia). Stimulation parameters are provided in Table 1.

\section{Assessment of headache frequency, severity and load}

Headache severity was evaluated on a verbal rating scale (VRS) for pain ( 0 no pain and 10 the worst pain imaginable). Headache frequency was described as the number of $\mathrm{CH}$ episodes per day. Headache load (HAL) is a composite score to simultaneously measure frequency, severity and duration of cluster headache episodes. It was calculated as $\sum$ (severity [verbal rating scale] $\mathrm{x}$ duration [in hours]) of all headache attacks experienced over a 2-week period ${ }^{17}$. These measures were assessed using headache diaries and were used as the clinical outcome measure.

\section{Neuropsychological Assessment}

Participants were evaluated within one month prior to the DBS procedure and one year or more post-operatively (mean 14 months \pm 4.5 ). The neuropsychological battery of tests was selected to be a comprehensive and assess various cognitive domains and involved assessment of global cognition, estimates of premorbid and current IQ, verbal and non-verbal memory, executive function, attention and language (for details please see $)^{2}$.

\section{Global Cognition and Intelligence}

The Mini Mental State Examination (MMSE) ${ }^{25}$ was used as a measure of global cognitive functioning. The National Adult Reading Test (NART) ${ }^{26}$ was used to obtain an estimate of premorbid intelligence quotient (IQ), and the Wechsler Abbreviated Scale of Intelligence (WASI) ${ }^{27}$ was administered to obtain an estimate of current Full Scale IQ. The NART assesses reading and vocabulary skills. The participants are asked to read aloud 50 irregular English words. The total 
error score is used to obtain an estimate of premorbid IQ. The WASI is an abbreviated scale to provide a measure of current intelligence quotient (IQ). It includes 4 subscales: vocabulary, similarities, block design, matrix reasoning. The vocabulary scale is indicative of verbal knowledge and verbal concept formation. Matrix Reasoning measures non-verbal skills of fluid and abstract reasoning. In the Short form of the WASI, the Vocabulary and Matrix Reasoning scores are used to obtain an estimation of the current Full Scale IQ.

\section{Verbal and Non-Verbal Memory}

The California Verbal Learning Test (CLVT-II) ${ }^{28}$ is a test of episodic verbal memory for words that provides data on immediate memory span, verbal learning, short/long term free and cued recall and delayed recognition of of 16 words, which belong to four semantic categories.

The Recognition Memory for Faces-short form (SRMF) ${ }^{29}$ was used to assess non-verbal recognition memory for faces. SRMF consists of two phases. In the first phase, 25 photographs of male faces are shown at the speed of 1 every 3 seconds. The participant is asked to indicate if they consider the face to be pleasant or not pleasant. In the second phase, 25 pairs of faces are shown and the participant is asked to identify which of the two faces had been previously presented. The score is the total number of correctly recognized faces.

\section{Executive Functions and Inhibition}

The Delis-Kaplan Executive Function System (D-KEFS) ${ }^{30}$ is a set of tests sensitive to verbal and nonverbal executive functions. In our study, the following subtests were used to assess executive function: the Trail Making Test (TMT), the phonemic, semantic and alternating categories Verbal fluency (VF) and the Colour-Word Interference Test (Stroop). In the D-KEFS verbal fluency the participant has 60 seconds to orally produce as many words as possible. There are three conditions: in the first condition (letter fluency) the participants are presented with a letter ( F, A and S) and asked to say as many words as possible starting with that letter. In the second phase (category fluency) the participants are asked to say as many words as possible in the category of animals and boy's names. In the third phase (switching) the participant is asked to say as many words as 
possible alternating between a fruit and furniture category. The score for each condition is the total number of correct items produced. Based on the original test designed by Stroop (1935), the DelisKaplan Colour-Word Interference test consists of four subtests. The "colour naming" (naming colour of ink of coloured rectangles) and "word reading" (reading colour words printed in black ink) subtests serve as control conditions. The main 'Stroop' test is the "inhibition" subtest which presents the names of colour words such as red, green and blue on the page printed in an incongruent colour of ink. For example, the word red is printed in blue ink. The patient has to name the colour of the ink as fast as they can. This requires inhibition of the more habitual and prepotent response of reading the word. Executive functions employed in this subtest are response inhibition, cognitive control and flexibility ${ }^{30}$. The final inhibition/switching condition also involves colour words printed in incongruent ink and requires switching between naming the colour of ink they are printed in and reading the word when the word is surrounded by a box. This condition has the additional requirement of switching between two task sets. The Trail Making Test includes 5 conditions ${ }^{30}$ :visual scanning, number sequencing, letter sequencing, number and letter switching and motor speed. In the visual scanning subtest the participants were shown letters and numbers and asked to strike off all " $3 \mathrm{~s}$ " presented in a group of letters and other numbers. In the number sequencing subtest the participants were asked to join all the numbers in progressive order ignoring the letters. In the letter sequencing subtest, the participants were asked to connect all the letters in alphabetical order and to ignore the numbers. In the number-letter switching subtest, the participants were asked to join numbers and letters in two alternating sequences of a number and then a letter (eg 1-A-2-B-3-C...). The motor speed test required the participants to be as quick as possible to trace dashed lines connecting circles. The executive functions believed to be important for the successful completion of this test are: behavioural regulation, cognitive flexibility and inhibition of perseverative responding.

\section{Attention}


The Paced Auditory Serial Addition Test (PASAT) ${ }^{31}$ involves presentation via a tape-recorder of a series of 31 numbers between 1 and 9. The patient is required to add each number read out to the immediately preceding one and say out their sum. Performance engages working memory and sustained attention. The percent correct is calculated.

\section{Depression and anxiety}

The Beck Depression Inventory (BDI-II) ${ }^{32}$ is a self-report inventory assessing the severity of depression with regard to the cognitive, affective, somatic, or behavioural symptoms. Scores range from 0 to 63, with higher scores denoting higher depression. The Hospital Anxiety and Depression Rating Scale (HADS) ${ }^{33}$ is a self-report measure assessing depression and anxiety. The sum of items in each subscale represents a total score indicating global anxiety (HADS-A) or depression (HADSD). On both Depression and Anxiety subscales scores range from 0 to 21, with higher scores indicating more severe depression or anxiety and scores above 11 considered 'caseness'.

\section{Hopelessness}

The Beck Hopelessness Scale (BHS) ${ }^{34}$ is a self-report measure of three major aspects of hopelessness: feelings about the future, loss of motivation, and expectations. The sum of items ranges from 0 to 20, and higher scores are indicative of higher hopelessness.

\section{Apathy}

The Starkstein Apathy Scale (SAS) was used to assess reduction of interest, motivation, affective responsivity, and engagement in goal-directed behaviours. The SAS consists of 14 items that are answered on a four point Likert scale (scores: 'not at all', 'slightly', 'some', 'a lot'). The total scores range from 0-42 and greater scores indicate more severe apathy ${ }^{35}$.

\section{Pain experience and behaviour}

The McGill Pain Questionnaire (MPQ) ${ }^{36}$ contains 78 pain descriptors assigned to three categories of pain qualities: sensory, affective, and evaluative. There is also a miscellaneous category of painrelated words. The Pain Behaviour Checklist (PBC) ${ }^{37}$ is a self-report assessment to quantify three classes of pain behaviours: help seeking, avoidance, and complaint. 


\section{Quality of Life}

The Short Form-36 (SF-36) is a 36 item questionnaire which measures Quality of Life (QoL) across eight domains (physical and social functioning, physical and emotional role limitations, mental health, energy, pain, and general health perceptions). Eight different subscores, and a physical and mental summary score can be derived. The maximum score ranges from 0 (lowest or worst possible level of functioning) to 100 (indicates the best possible health state).

\section{Statistical analysis}

All data were analysed using the computing environment $\mathrm{R}^{38}$. The data for all tests were first assessed for presence of outliers, the parametric assumptions of normality (Shapiro-Wilk Test), and homogeneity of variance (Bartlett's Test). When the assumptions for parametric analysis were met, paired samples t-tests were used to compare cognitive, mood, pain and quality of life measurements before and after VTA-DBS. When the assumptions were violated, nonparametric Wilcoxon signed ranks test was used and the median was given instead of the mean. To protect against Type I Error, for each outcome measure domain (Cognitive, Mood, Pain and Quality of Life) a Bonferroni correction was completed and applied. Thus the corrected $\mathrm{p}$ value was used for determination of significance of the results in each domain. The corrected p-value for the measures of Cognitive function is $\mathrm{p}=.001$ (that is $\alpha_{\text {altered }}=.05 / 30=.001$ ). For the measures of Mood the corrected $\mathrm{p}=$ .01 ; for Pain-related Behaviours it is $\mathrm{p}=.006$; for the Quality of life measure it is $\mathrm{p}=.006$. Furthermore, to determine if any of the post-operative changes were statistically reliable, we calculated the reliable change index $(\mathrm{RCI})$. RCI verifies if variation in a score is statistically strong and reliable ${ }^{39}$. The formula for calculating it is:

$$
\frac{X_{2}-X_{1}}{S_{\text {diff }}}
$$

Mean of post operation score minus mean of pre operation score divided by the standard error of the difference. The formula for calculating the standard error of difference is: 


$$
S_{\text {diff }}=\sqrt{ } 2\left(S_{1} \sqrt{1}-r_{x x}\right)^{2}
$$

The RCI cut off is $1.96, \mathrm{RCI}<-1.96$ are considered a reliable decline, RCI $>+1.96$ are considered a reliable improvement, RCI between -1.96 and +1.96 are not considered as reflecting reliable change. We also calculated the confidence intervals of reliable changes for specific measures. The formula for calculating this is:

$$
X_{2}-X_{1}=S_{\text {diff }} \times \pm 1.96
$$

Based on this score the results are then regrouped into three classes representing the percentages of the participants with reliable decline, no change and reliable improvement.

\section{Results}

Headache frequency, severity and load before and after surgery

Headache frequency measured as the number of daily attacks was significantly reduced from 5 to 2 after VTA-DBS $(\mathrm{p}<.001)$. Indicating that there was a $52 \%$ overall improvement in headache frequency. Fifty five percent of patients had a reduction of $\geq 40 \%$ on the number of daily attacks after VTA-DBS. Headache severity assessed on the verbal rating scale (VRS) was significantly reduced from 10 to 7 after VTA-DBS $(\mathrm{p}<.001)$, indicating that there was a $30 \%$ overall improvement in headache severity. Forty percent of patients had a reduction of $\geq 30 \%$ on the VRS after VTA-DBS. Headache load (HAL) was significantly reduced after (Median = 177.00) compared to before VTA-DBS (Median=757.00), $\mathrm{p}<.005, \mathrm{r}=-.72$. Thirteen patients $(72 \%)$ had a reduction of $\geq 30 \%$ on the HAL score after VTA-DBS. Their measures of cognitive function, and mood, pain, disability, quality of life before and after surgery are shown respectively in Tables 2 and 3. 
Measures of global cognition and intelligence

Similar to the pre-operative MMSE score, the group median MMSE score at follow-up was also within the normal range. With the corrected $\mathrm{p}$ value of .001 , the differences between pre and post DBS surgery in the MMSE scores were not significant. There were no significant differences between pre- and post-DBS measures of the WASI estimate of current Full Scale IQ. There were no significant differences between pre- and postoperative measures of estimated premorbid IQ (NART), and between current and estimated premorbid IQ either before $(\mathrm{p}=.57)$ or after $(\mathrm{p}=.38)$ surgery.

\section{Executive Functions and inhibition}

The pre- and post-DBS scores on the measures of executive function on the Trail-making, the Stroop and the verbal fluency tasks are presented in Table 2. The scaled score for the total correct category switches on the alternating category VF test was lower after $(\mathrm{M}=8.33, \mathrm{SD}=1.97)$ than before $(M=10.17, S D=4.09)$ surgery, but this change was not significant at the corrected $\mathrm{p}$ value of $\mathrm{p}<.001$. The reliable change index $(\mathrm{RCI}=-0.59)$ also indicated that this was not a reliable change. There were no other significant differences between pre- and post-measures on the D-KEFS subtests of the Trail-Making Test, verbal fluency or Stroop Colour-Word interference.

\section{Memory}

As evident from Table 2, there were no significant differences between pre and post VTA-DBS measures derived from the CVLT-II or on the short Recognition Memory for Faces (all p>.001). Pain experience and behaviour before and after VTA-DBS (Table 3)

After Bonferroni correction there were no significant differences on the Pain-Behaviour Checklist $(\mathrm{PBC})$ total scores after $(\mathrm{M}=21.78, \mathrm{SD}=15.09)$ compared to before $(\mathrm{M}=27.60, \mathrm{SD}=11.02), \mathrm{t}(14)$ $=2.17, \mathrm{p}=.047, \mathrm{r}=.50$ surgery. However, the PBC Help Seeking behaviours were significantly reduced after $(M=2.61, S D=1.50)$ compared to before $(M=4.00, S D=1.00), t(14)=4.36, p<$ $.001, r=.76$ surgery, suggesting less engagement in help-seeking behaviours for their $\mathrm{CH}$ pain 
from patients after surgery (Figure $1 \mathrm{~A})$. The reliable change index $(\mathrm{RCI}=-4.43)$ indicates that this change is reliable. At post-surgical follow-up, a larger proportion of the participants showed improvement $(80 \%)$ than no change $(13 \%)$ or decline $(6 \%)$ in help-seeking behaviours. The avoidance and complaints subscales of the PBC were not significantly altered by DBS. There were no statistically significant differences between pre- $(\mathrm{M}=46.06, \mathrm{SD}=19.35)$ and post-surgery $(\mathrm{M}=$ 50.28, $\mathrm{SD}=15.97), \mathrm{t}(12)=0.10, \mathrm{p}=0.92$ scores on the McGill Pain Questionnaire or any of the sensory, evaluative, affective or miscellaneous subscores (see Table 3).

Measures of mood and quality of life before and after VTA-DBS (Table 3)

After Bonferroni correction, on the HADS-A scale, self-reported anxiety levels were significantly reduced after $(\mathrm{M}=8.00, \mathrm{SD}=4.51)$ compared to before VTA-DBS $(\mathrm{M}=11.94, \mathrm{SD}=4.36), \mathrm{t}(17)=$ 5.43, $\mathrm{p}<.001, \mathrm{r}=.79$. (Figure $1 \mathrm{~B})$. The reliable change index $(\mathrm{RCI}=-2.86)$ indicated a reliable reduction of anxiety. At follow up, $61 \%$ of patients had a reliable reduction of anxiety, 39\% had no change and no patient had an increase of anxiety. While the means suggest some improvement in depression after surgery, there were no statistically significant differences between pre $(M=23.11$, $\mathrm{SD}=11.88)$ and post $(\mathrm{M}=20.55, \mathrm{SD}=12.16), \mathrm{t}(17)=1.50, \mathrm{p}=.15$ surgery measures of the Beck Depression Inventory. Similarly, there were no statistically significant differences between pre $(\mathrm{M}=11.61, \mathrm{SD}=5.09)$ and post $(\mathrm{M}=10.67, \mathrm{SD}=5.82)$ surgery scores on the HADS-D, $\mathrm{t}(17)=1.22, \mathrm{p}$ $=.23$. Scores on the Hopelessness Scale and the Starkstein Apathy Scale were not altered by surgery (see Table 3 for the pre and post-operative means). On the SF36 measure of quality of life, the score on the Social Role Functioning subscale was higher after (Median=43.75) compared to before $($ Median $=25.00)$, indicating better social functioning of patients after VTA-DBS, however after Bonferroni correction this was not significant. Similarly, on the SF36, the score on the emotional wellbeing subscale was higher after $(M=55.44)$ compared to before $(M=47.06)$, surgery, indicating greater emotional wellbeing of patients after VTA-DBS, however after Bonferroni correction the improvement was not significant. Nevertheless, at follow up $46 \%$ of the patients had better social functioning. 


\section{Correlational Analysis}

Pearson correlational analyses were performed, to explore the relationship between the change scores in Headache Load (before and after VTA-DBS) and change scores in mood, pain experience and behaviour, and quality of life (listed in Table 3) and cognitive measures (listed in Table 2). Variations in anxiety levels correlated positively and significantly with changes in Headache Load $(\mathrm{r}=0.57, \mathrm{p}<.01)$. None of the other correlations were of a notable magnitude or significant.

\section{Discussion}

A previous clinical study provided evidence for the positive impact of VTA-DBS on CH severity and frequency ${ }^{24}$. In particular Akram and colleagues demonstrated a significant decrease in headache frequency, severity and load after surgery, with an associated relative decrease of triptan medication intake. However, to our knowledge, the influence of VTA-DBS on measures of cognition, mood, pain experience and behaviour, and quality of life has not been previously examined in detail, which was the aim of this study. We found that VTA-DBS produced clinical benefits on $\mathrm{CH}$ frequency, severity and load and produced no adverse effects on cognitive function, but was associated with significant decrease of anxiety, and significant improvement of pain-related help-seeking behaviours indicating better coping with pain after surgery.

Akram and colleagues defined the target area for the DBS electrode as the ventral tegmental area (VTA). The VTA, is the most anterior aspect of the midbrain tegmentum and encloses distinct types of neuronal cells. However, it is mainly distinguished by its abundant number of dopamine (DA) neurons.Together with the substantia nigra, the VTA is considered to be a main dopaminergic territory in the brain ${ }^{40}$. Projections of dopaminergic neurons start from the VTA to reach various regions of the cortex through two main routes: the mesocortical and the mesolimbic dopaminergic pathways. The former transmits information from the VTA to prefrontal, orbitofrontal and cingulate 
cortices. The dorsolateral prefrontal cortex (DLPFC) ${ }^{41,42}$, receives dopaminergic signals from VTA and it has been charachterized to be an important node for a range of cognitive funtions such as working memory ${ }^{43,44}$, behavioural flexibility and inhibitory control of willed actions ${ }^{45,46}$ aspects of social cognition, emotional regulation, memory retrieval ${ }^{47}$ and implicit temporal processing ${ }^{48-50}$. Thus, dopaminergic projections to the forebrain, including the frontal lobes and the dorsal and ventral striatum, constitute a fundamental part of the neural circuits underlying a variety of cognitive and executive functions. A recent patch clamp electrophysiology study in rats demonstrated the functional importance of VTA terminals to driving intrinsic inhibition in the PFC 51. The mesolimbic path projects from the VTA to reach different limbic areas. In particular there are numerous connections with the nucleus accumbens (NAc). Among the functions that have been attributed to this mesolimbic dopaminergic circuit there is the regulation of the reward system ${ }^{52}$. It has been shown that VTA dopamine neurons are responsive to anticipation of time of reward, unpleasant or new stimuli ${ }^{53-55}$. Recently, a computational model of the afferents to the VTA that replicates many of the experimental observations has been proposed ${ }^{56}$. In light of the connectivity of the VTA with the prefrontal cortex, in the present study, we administered subtests of the DelisKaplan Executive Function System (D-KEFS) ${ }^{30}$ a set of tests to assess verbal and nonverbal executive functions before and after VTA-DBS to test for any possible effects on 'prefrontal' executive functions. The results showed that VTA-DBS surgery did not significantly alter any of the measures of executive function included.

\section{Effect of VTA-DBS on cognitive function}

Cognitive function in $\mathrm{CH}$ has been examined in previous studies. While some have reported impairments in verbal memory in $\mathrm{CH}^{57}$, others did not find any significant differences in memory or executive function in $\mathrm{CH}$ patients relative to healthy controls ${ }^{58}$. In a previous study by our group, Torkamani et al (2013) found no impairment in IQ, verbal memory or executive functions in patients with chronic or episodic $\mathrm{CH}$ relative to matched healthy controls and only deficits in 
working memory and self-reported cognitive failures were documented. Other evidence has suggested that cognitive performance in $\mathrm{CH}$ is altered only during headache attacks ${ }^{59}$, due to the fact that pain can also make demands on attentional resources ${ }^{60}$. In this study, we examined the impact of VTA-DBS on global cognitive function and more specific cognitive domains of intelligence, executive function, memory and attention. The effective site of the DBS for $\mathrm{CH}$ in the ipsilateral hypothalamus was shown to have high probability connectivity to the frontal cortex ${ }^{21,61}$. In a similar vein, it is likely that stimulation through electrode contacts located in the VTA modulates distant neuronal dynamics through its electrical and chemical influences. However, we observed no significant cognitive changes in executive function after VTA-DBS (see Table 2). Previous research ${ }^{2}$ showed no major deficits on tests of executive function in $\mathrm{CH}$ patients relative to healthy controls, including the Stroop and verbal fluency tests also employed here, and the results of the present study provide unique data that indicate that surgical implantation of electrodes and chronic stimulation of the VTA is generally safe from a cognitive perspective.

\section{Effect of VTA-DBS on pain experience and behaviour, mood and quality of life}

The impact of $\mathrm{CH}$ on mood, daily functioning and QoL has been measured in a number of previous studies. These have reported that patients with $\mathrm{CH}$ had elevated levels of depression, suicidal ideation, anxiety, hopelessness, and increased disability and poorer QoL relative to healthy controls 2,11,57,62-64. Anxiety symptoms, as measured by the HAD-A, significantly improved after DBS-VTA surgery. The RCI confirmed these changes showing that one year postoperatively the majority of patients showed improvement $(61 \%)$ and none of the participants showed worsening of anxiety after VTA-DBS. It is difficult to determine whether this beneficial effect on anxiety is generated by VTA stimulation directly altering the activity in the mesolimbic circuits, or indirectly because VTA-DBS reduced headache frequency and severity. In current experimental paradigms, anxiety has been operationalized as an emotional response to potential threats. VTA is a crucial region in the anxiety circuit and receives both excitatory glutamatergic and inhibitory GABAergic inputs 
from the ventral bed nucleus stria terminalis (vBNST). The current concept is that two parallel vBNST-to-VTA pathways mediate either anxiogenic or anxiolytic behavioural response. Specifically, activation of glutamatergic vBNST inputs to the VTA increased anxiety and induced avoidance, whereas activation of GABAergic inputs enhanced anxiolytic effects ${ }^{65}$. Therefore, a possible explanation for the reduction of anxiety following VTA-DBS might be that the stimulation disrupts the glutamatergic inputs from vBNST. Another possibility is that stimulating VTA leads to a similar effect to the GABAergic inputs that produce anxiolytic effects. A second explanation is that anxiety reduction is secondarily related to reduced headache frequency and severity. Further work is required to establish the mechanisms of the reduction of anxiety following VTA-DBS.

On the Pain Behaviour Checklist, the help-seeking behaviours were significantly reduced after VTA-DBS compared to before surgery, suggestive of better adjustment and coping with pain. The RCI endorsed these changes showing that more patients showed improvement (80\%) than deterioration $(13 \%)$ or no change $(6 \%)$ on the Pain Behaviour Checklist after VTA-DBS. However, the changes in the severity or qualitative aspects of pain experience on the McGill Pain Questionnaire were not altered following VTA-DBS. Hopelessness and apathy were also not significantly altered by surgery. Depression, showed a trend toward reduction (pre-operative, $M=23.11$, post-operative, $M=20.55$ ) but was not significantly changed by surgery. With VTA-DBS, the social role functioning and emotional wellbeing components of QoL on the SF36 improved, albeit non-significantly after Bonferroni correction.

A major limitation of this study is the small sample size. However given the infrequency of the VTA-DBS procedure for refractory $\mathrm{CH}$ the current sample is satisfactory. A second limitation is that multiple comparisons were conducted which increases the risk of type 1 error. To overcome this limitation we conducted a Bonferroni correction. Despite these limitations, the study provides new information about DBS-VTA safety and efficacy. In conclusion, VTA-DBS, did not produce any adverse cognitive effects on the measures of cognition assessed and was associated with 
significant improvement of anxiety and help-seeking pain-related behaviours and non-significant improvement of the social role functioning and emotional wellbeing aspects of quality of life one year after surgery in our sample of $\mathrm{CH}$ patients.

\section{Article Highlights:}

- Deep brain stimulation of the ventral tegmental area (VTA-DBS) in patients with medically refractory chronic cluster headache $(\mathrm{CH})$ did not induce any significant adverse cognitive effects.

- There was significant improvement of anxiety symptoms after VTA-DBS.

- There was significant reduction of help seeking pain-related behaviours after VTA-DBS suggestive of better adjustment and coping with pain after surgery. 



\section{References}

1. Headache Classification Subcommittee of The International Headache Society. The International Classification of Headache Disorders 3rd edition (beta version). Cephalagia 2013; 33: 629-808.

2. Torkamani M, Ernst L, Cheung LS, et al. The Neuropsychology of Cluster Headache: Cognition, Mood, Disability, and Quality of Life of Patients With Chronic and Episodic Cluster Headache. Headache J Head Face Pain 2015; 55: 287-300.

3. Bussone G. Cluster headache: From treatment to pathophysiology. Neurol Sci 2008; 29: 1-6.

4. Tommaso M De, Vecchio E. Primary headaches and trigeminal neuralgia : neuropathic pain yes or not? Evidences from neurophysiological procedures. Expert Rev Neurother 2013; 13: 1031-1039.

5. Holland PR. Modulation of trigeminovascular processing: Novel insights into primary headache disorders. Cephalalgia 2009; 29: 1-6.

6. Holle D, Obermann M. Cluster headache and the hypothalamus: Causal relationship or epiphenomenon? Expert Review of Neurotherapeutics 2011; 11: 1255-1263.

7. Leone M, Bussone G. Pathophysiology of trigeminal autonomic cephalalgias. The Lancet Neurology 2009; 8: 755-764.

8. Nardone R, Ausserer H, Bratti A, et al. Trigemino-cervical reflex abnormalities in patients with migraine and cluster headache. Headache 2008; 48: 578-585.

9. Waldenlind E, Sjöstrand C. Pathophysiology of cluster headache and other trigeminal autonomic cephalalgias. Epub ahead of print 2010. DOI: 10.1016/S0072-9752(10)97033-4.

10. Goadsby PJ. Trigeminal Autonomic Cephalalgias. Contin Lifelong Learn Neurol 2012; 18: 883-895.

11. Donnet A, Lanteri-Minet M, Guegan-Massardier E, et al. Chronic cluster headache: a French clinical descriptive study. J Neurol Neurosurg Psychiatry 2007; 78: 1354-8.

12. Leone M, Bussone G. A Review of Hormonal Findings in Cluster Headache. Evidence for Hypothalamic Involvement. Cephalalgia 1993; 13: 309-317.

13. Waldenlind E, Gustafsson S. Prolactin in Cluster Headache: Diurnal Secretion, Response to Thyrotropin-Releasing Hormone, and Relation to Sex Steroids and Gonadotropins. Cephalalgia 1987; 7: 43-54.

14. May A, Bahra A, Büchel C, et al. Hypothalamic activation in cluster headache attacks. Lancet 1998; 352: $275-278$.

15. May A, Bahra A, Büchel C, et al. Functional magnetic resonance imaging in spontaneous attacks of SUNCT: short-lasting neuralgiform headache with conjunctival injection and tearing. Ann Neurol 1999; 46: 791-4.

16. Cutrer FM, Charles A. The neurogenic basis of migraine. Headache 2008; 48: 1411-1414.

17. Akram H, Miller S, Lagrata S, et al. Ventral tegmental area deep brain stimulation for refractory chronic cluster headache. Neurology 2016; 86: 1676-1682.

18. Fontaine D, Lanteri-Minet M, Ouchchane L, et al. Anatomical location of effective deep brain stimulation electrodes in chronic cluster headache. Brain 2010; 133: 1214-1223.

19. Matharu MS, Zrinzo L. Deep brain stimulation in cluster headache: Hypothalamus or midbrain 
tegmentum? Current Pain and Headache Reports 2010; 14: 151-159.

20. Goadsby PJ, Lipton RB. A review of paroxysmal hemicranias, SUNCT syndrome and other shortlasting headaches with autonomic feature, including new cases. Brain 1997; 120: 193-209.

21. Akram H, Miller S, Lagrata S, et al. Optimal deep brain stimulation site and target connectivity for chronic cluster headache. Neurology 2017; 89: 2083-2091.

22. May A, Leone M, Áfra J, et al. EFNS guidelines on the treatment of cluster headache and other trigeminal-autonomic cephalalgias. Eur J Neurol 2006; 13: 1066-1077.

23. Leone M, Franzini A, Bussone G. Stereotactic Stimulation of Posterior Hypothalamic Gray Matter in a Patient with Intractable Cluster Headache. N Engl J Med 2001; 345: 1428-1429.

24. Akram H, Miller S, Lagrata S, et al. Ventral tegmental area deep brain stimulation for refractory chronic cluster headache. Neurology 2016; 86: 1676-1682.

25. Folstein MF, Folstein SE, McHugh PR, et al. \&quot;Mini-mental state\&quot;. A practical method for grading the cognitive state of patients for the clinician. J Psychiatr Res 1975; 12: 189-98.

26. Nelson H, Willison J. National Adult Reading Test (NART)., http://www.academia.edu/download/31611053/NART_MANUAL.pdf (1991, accessed 6 June 2017).

27. Wechsler D. Wechsler abbreviated scale of intelligence, https://scholar.google.com/scholar?q=Wechsler+D.+Wechsler+Abbreviated+Scale+of+Intelligence.+ $\mathrm{New}+$ York\% 252C+NY\%253A+Psychological+Corporation\%253B $+1999 . \& b t n G=\& h l=i t \& a s \_s d t=0$ $\% 252 \mathrm{C} 5$ (1999, accessed 6 June 2017).

28. Delis D, Kramer J, Kaplan E, et al. CVLT-II: California verbal learning test: adult version, https://scholar.google.com/scholar?q=Delis+DC\%252C+Kramer+J\%252C+Kaplan+E\%252C+Ober+ BA.+California+Verbal+Learning+Test.+New+York\%252C+NY\%253A+Psychological+Corporatio n\%253B+2000\&btnG=\&hl=it\&as_sdt=0\%252C5 (2000, accessed 6 June 2017).

29. Warrington E. The Camden memory tests manual, https://books.google.com/books?hl=it\&lr=\&id=RlPbPAMQuScC\&oi=fnd\&pg=PA1\&dq=warrington $+1996+$ camden\&ots=OuLfqyc-A8\&sig=jNzq0hrI55vaMetqEltv3zZ1Pio (1996, accessed 6 June 2017).

30. Delis D, Kaplan E, Kramer J. Delis-Kaplan executive function system (D-KEFS).

31. Gronwall DMA. Paced Auditory Serial-Addition Task: A Measure of Recovery from Concussion. Percept Mot Skills 1977; 44: 367-373.

32. Beck AT, Steer RA, Ball R, et al. Comparison of Beck Depression Inventories -IA and -II in psychiatric outpatients. J Pers Assess 1996; 67: 588-97.

33. Zigmond A, Snaith R. The hospital anxiety and depression scale. Acta Psychiatr Scand, http://onlinelibrary.wiley.com/doi/10.1111/j.1600-0447.1983.tb09716.x/full (1983, accessed 6 June 2017).

34. Beck A, Steer R. Beck hopelessness scale, http://www.incamresearch.ca/content/beck-hopelessnessscale (1988, accessed 6 June 2017).

35. Starkstein SE, Mayberg HS, Preziosi TJ, et al. Reliability, validity, and clinical correlates of apathy in Parkinson's disease. J Neuropsychiatry Clin Neurosci 1992; 4: 134-139.

36. Melzack R. The McGill Pain Questionnaire: major properties and scoring methods. Pain, http://www.sciencedirect.com/science/article/pii/0304395975900445 (1975, accessed 6 June 2017).

37. Philips H. Avoidance behaviour and its role in sustaining chronic pain. Behav Res Ther, 
http://www.sciencedirect.com/science/article/pii/0005796787900052 (1987, accessed 6 June 2017).

38. Team R. Vienna, Austria: R Foundation for Statistical Computing; 2013. R: A language and environment for statistical computing. -07-0, URL http//www R-project org.

39. Jacobson NS, Truax P. Clinical significance: A statistical approach to defining meaningful change in psychotherapy research. J Consult Clin Psychol 1991; 59: 12-19.

40. Björklund A, Dunnett SB. Dopamine neuron systems in the brain: an update. Trends Neurosci 2007; 30: 194-202.

41. Seamans JK, Yang CR. The principal features and mechanisms of dopamine modulation in the prefrontal cortex. Prog Neurobiol 2004; 74: 1-58.

42. Szczepanski SM, Knight RT. Insights into human behavior from lesions to the prefrontal cortex. Neuron 2014; 83: 1002-18.

43. D'Esposito M. From cognitive to neural models of working memory. Philos Trans $R$ Soc Lond B Biol Sci 2007; 362: 761-72.

44. Goldman-Rakic PS. Regional and cellular fractionation of working memory. Proc Natl Acad Sci U S A 1996; 93: 13473-80.

45. Casey BJ, Castellanos FX, Giedd JN, et al. Implication of right frontostriatal circuitry in response inhibition and attention-deficit/hyperactivity disorder. J Am Acad Child Adolesc Psychiatry 1997; 36: $374-83$.

46. Jahanshahi M. WILLED ACTION AND ITS IMPAIRMENTS. Cogn Neuropsychol 1998; 15: 483533.

47. Gilbert SJ, Burgess PW. Executive function. Curr Biol 2008; 18: R110-4.

48. Tomassini A, Ruge D, Galea J, et al. The Role of Dopamine in Temporal Uncertainty. J Cogn Neurosci 2016; 28: 96-110.

49. Vallesi A, Shallice T, Walsh V. Role of the Prefrontal Cortex in the Foreperiod Effect: TMS Evidence for Dual Mechanisms in Temporal Preparation. Cereb Cortex 2006; 17: 466-474.

50. Vallesi A, McIntosh AR, Alexander MP, et al. FMRI evidence of a functional network setting the criteria for withholding a response. Neuroimage 2009; 45: 537-548.

51. Buchta WC, Mahler S V, Harlan B, et al. Dopamine terminals from the ventral tegmental area gate intrinsic inhibition in the prefrontal cortex. Physiol Rep 2017; 5: e13198.

52. Berridge KC, Robinson TE. What is the role of dopamine in reward: hedonic impact, reward learning, or incentive salience? Brain Res Rev 1998; 28: 309-369.

53. Redgrave P, Gurney K, Reynolds J. What is reinforced by phasic dopamine signals? Brain Res Rev 2008; 58: 322-339.

54. Horvitz JC. Mesolimbocortical and nigrostriatal dopamine responses to salient non-reward events. Neuroscience 2000; 96: 651-6.

55. Schultz W, Dayan P, Montague PR. A neural substrate of prediction and reward. Science 1997; 275: 1593-9.

56. Vitay J, Hamker FH. Timing and expectation of reward: a neuro-computational model of the afferents to the ventral tegmental area. Front Neurorobot 2014; 8: 4.

57. Jorge RE, Leston JE, Arndt S, et al. Cluster headaches: association with anxiety disorders and 
memory deficits. Neurology 1999; 53: 543-7.

58. Sinforiani E, Farina S, Mancuso A, et al. Analysis of higher nervous functions in migraine and cluster headache. Funct Neurol; 2: 69-77.

59. Evers S. Cognitive processing in cluster headache. Curr Pain Headache Rep 2005; 9: 109-112.

60. Eccleston C, Crombez G. Pain demands attention: A cognitive-affective model of the interruptive function of pain. Psychol Bull 1999; 125: 356-366.

61. Owen SLF, Green AL, Davies P, et al. Connectivity of an effective hypothalamic surgical target for cluster headache. J Clin Neurosci 2007; 14: 955-960.

62. Jurgens TP, Gaul C, Lindwurm A, et al. Impairment in episodic and chronic cluster headache. Cephalalgia 2011; 31: 671-682.

63. D'Amico D, Usai S, Grazzi L, et al. Quality of life and disability in primary chronic daily headaches. Neurol Sci 2003; 24 Suppl 2: S97-100.

64. Liang J-F, Chen Y-T, Fuh J-L, et al. Cluster headache is associated with an increased risk of depression: a nationwide population-based cohort study. Cephalalgia 2013; 33: 182-9.

65. Jennings JH, Sparta DR, Stamatakis AM, et al. Distinct extended amygdala circuits for divergent motivational states. Nature 2013; 496: 224-228. 

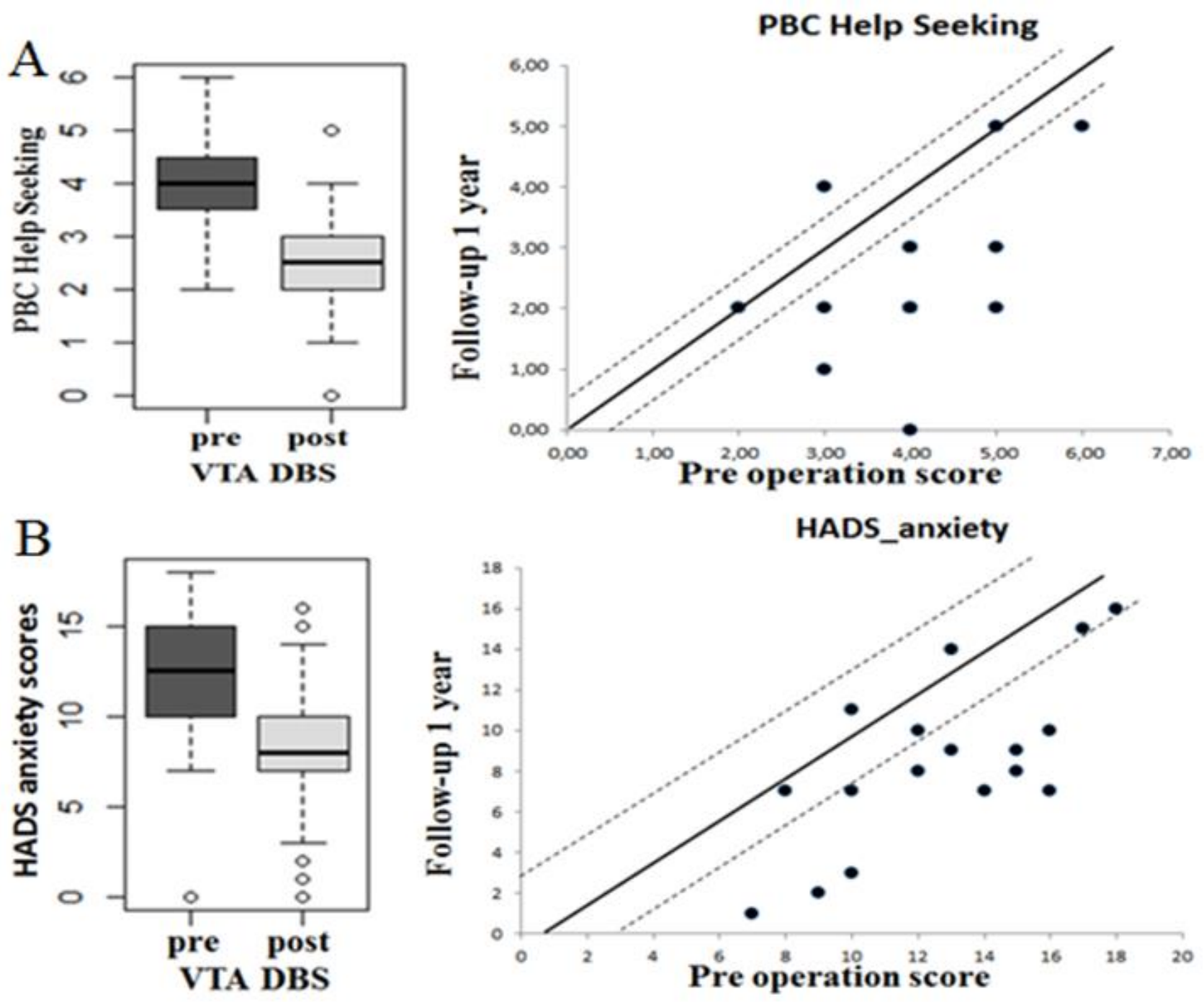

\section{Figure 1:}

Boxplots showing the distribution of values before and after VTA-DBS for (A) Pain Behaviour Checklist Help Seeking Behaviours, (B) Hospital Anxiety \& Depression Scale, Anxiety subscale. Black dots represent patients' raw scores. The solid line represents zero change. Values above the top dotted line represent a reliable increase in scores from baseline to follow-up after VTA-DBS. Values below the lower dotted line represent a reliable decrease in scores from baseline to followup after VTA-DBS, values between the dotted lines represent no reliable change. 
Table 1. Demographic and clinical details and deep brain stimulation (DBS) parameters of the patients with cluster headache.

\begin{tabular}{|c|c|c|c|c|c|c|c|c|c|c|}
\hline ID & $\begin{array}{c}\text { Age } \\
\text { (years) }\end{array}$ & Gender & $\begin{array}{c}\text { Duration } \\
\text { (years) }\end{array}$ & $\begin{array}{c}\text { electrode_positions } \\
\text { (hemisphere) }\end{array}$ & $\begin{array}{c}\text { DBS } \\
\text { intensity_left } \\
\text { (V) }\end{array}$ & $\begin{array}{c}\text { DBS } \\
\text { intensity_right } \\
\text { (V) }\end{array}$ & $\begin{array}{c}\text { DBS } \\
\text { frequency_left } \\
(\mathrm{Hz})\end{array}$ & $\begin{array}{c}\text { DBS } \\
\text { frequency_right } \\
(\mathrm{Hz})\end{array}$ & $\begin{array}{c}\text { DBS pulse } \\
\text { width_left } \\
(\mu S)\end{array}$ & $\begin{array}{c}\text { DBS pulse } \\
\text { width_right } \\
(\mu \mathrm{S})\end{array}$ \\
\hline 1 & 46 & $\bar{M}$ & 7 & right & - & 2.1 & - & 185 & - & 60 \\
\hline 2 & 49 & $\mathrm{M}$ & 25 & left & 0.9 & - & 185 & - & 60 & - \\
\hline 3 & 41 & F & 4 & bilateral & 3.0 & 3.0 & 185 & 185 & 60 & 60 \\
\hline 4 & 58 & M & 14 & left & 3.0 & - & 130 & - & 60 & - \\
\hline 5 & 42 & F & 21 & bilateral & 2.2 & 2.5 & 185 & 185 & 60 & 60 \\
\hline 6 & 67 & M & 14 & right & - & 1.0 & - & 185 & - & 60 \\
\hline 7 & 67 & M & 15 & left & 1,5 & - & 185 & - & 60 & - \\
\hline 8 & 33 & $\mathrm{M}$ & 17 & right & - & 2.7 & - & 185 & - & 60 \\
\hline 9 & 49 & F & 11 & left & 1.5 & - & 185 & - & 60 & - \\
\hline 10 & 43 & M & 28 & right & - & 3.0 & - & 185 & - & 60 \\
\hline 11 & 37 & M & 23 & right & - & 3.0 & - & 185 & - & 60 \\
\hline 12 & 39 & M & 13 & bilateral & 3.0 & 1.5 & 185 & 185 & 60 & 60 \\
\hline 13 & 41 & M & 15 & left & 3.0 & - & 185 & - & 60 & - \\
\hline 14 & 53 & M & 22 & left & 3.2 & - & 185 & & 60 & - \\
\hline 15 & 59 & M & 20 & bilateral & 4.0 & 4.0 & 185 & 185 & 60 & 60 \\
\hline 16 & 48 & M & 15 & right & - & 3.5 & - & 185 & - & 60 \\
\hline 17 & 45 & M & 5 & bilateral & 3.0 & 3.0 & 185 & 185 & 60 & 60 \\
\hline \multirow[t]{2}{*}{18} & 56 & F & 12 & left & 3.5 & - & 185 & - & 60 & - \\
\hline & 46.73 & $\begin{array}{l}\mathrm{M}=78 \% \\
\mathrm{~F}=22 \%\end{array}$ & 11.94 & $\begin{array}{c}\text { Right }=6 \\
\text { Left }=7 \\
\text { Bilateral }=5\end{array}$ & & & & & & \\
\hline
\end{tabular}


Table 2. Means and standard deviations for the measures of cognitive function before and after VTA-DBS and reliable change indices

(RCI) showing percent with decline, no change and improvement on the cognitive measures.

\begin{tabular}{|c|c|c|c|c|c|c|c|c|}
\hline Measure & Sample & Pre-operative & Post-operative & $\begin{array}{l}p= \\
\text { correct } \\
\text { ed } \\
p<.001\end{array}$ & $\begin{array}{c}\text { Decline } \\
\%\end{array}$ & $\begin{array}{c}\text { No } \\
\text { Change } \\
\%\end{array}$ & $\begin{array}{c}\text { Improve } \\
\%\end{array}$ & $\begin{array}{c}\text { Overall RCI } \\
\text { Improvement }>1.96 \\
\text { Decline }<1.96 \\
\text { No change }- \\
1.96<\text { RCI }<1.96 \\
\end{array}$ \\
\hline \multicolumn{9}{|l|}{ Global Cognition } \\
\hline Mini Mental State Examination & 18 & $29.11 \pm 1.53$ & $28.27 \pm 1.45$ & .03 & 55.5 & 33.3 & 11.1 & -1.72 \\
\hline \multicolumn{9}{|l|}{ Measures of Intelligence } \\
\hline WASI-full scale IQ & 18 & $106.72 \pm 14.93$ & $103.83 \pm 15.43$ & .07 & 11.1 & 88.9 & 0.0 & -0.33 \\
\hline National Adult Reading Test & 18 & $108.94 \pm 8.79$ & $109.19 \pm 4.96$ & .72 & 12.5 & 68.7 & 18.7 & 0.18 \\
\hline \multicolumn{9}{|l|}{ California Verbal Learning Test-II } \\
\hline Trial 1 & 18 & $6.17 \pm 2.12$ & $6.05 \pm 1.98$ & .97 & 27.8 & 50.0 & 22.2 & -0.17 \\
\hline Trial 1-5 & 18 & $48.78 \pm 12.60$ & $48.06 \pm 11.95$ & .74 & 33.3 & 38.9 & 27.8 & -0.30 \\
\hline Short delay-free recall & 18 & $9.47 \pm 3.41$ & $9.00 \pm 3.97$ & .50 & 11.8 & 82.4 & 5.9 & -0.33 \\
\hline Short delay-cued recall & 18 & $10.76 \pm 3.15$ & $10.11 \pm 3.30$ & .37 & 35.3 & 52.9 & 11.8 & 0.53 \\
\hline Long delay-free recall & 18 & $9.71 \pm 3.06$ & $9.28 \pm 3.54$ & .41 & 23.5 & 70.6 & 5.9 & -0.37 \\
\hline Long delay-cued recall & 18 & $10.71 \pm 3.02$ & $10.50 \pm 3.29$ & .85 & 23.5 & 64.7 & 11.8 & -0.12 \\
\hline Recognition-hits & 18 & $13.41 \pm 3.24$ & $14.94 \pm 8.34$ & .96 & 29.4 & 47.1 & 23.5 & 1.60 \\
\hline Recognition-false positives & 18 & $1.53 \pm 1.84$ & $2.61 \pm 2.61$ & .13 & 11.8 & 58.9 & 29.4 & 1.85 \\
\hline \multicolumn{9}{|c|}{ Warrington Short Recognition Memory for Faces } \\
\hline Total correct responses ( $\max 25)$ & 18 & $22.82 \pm 2.30$ & $22.83 \pm 2.20$ & .95 & 11.8 & 70.6 & 17.6 & 0.00 \\
\hline \multicolumn{9}{|l|}{$D$-KEFS measures of executive function } \\
\hline \multicolumn{9}{|l|}{ TRAIL MAKING TEST scaled scores } \\
\hline Visual Scanning & 18 & $9.11 \pm 1.99$ & $8.82 \pm 3.06$ & .58 & 29.4 & 52.9 & 17.6 & -0.55 \\
\hline Number sequencing & 18 & $9.50 \pm 2.04$ & $10.35 \pm 2.26$ & .07 & 11.8 & 47.1 & 41.2 & 1.17 \\
\hline Letter sequencing & 18 & $9.10 \pm 3.39$ & $9.53 \pm 3.16$ & .33 & 5.9 & 58.8 & 35.3 & 0.37 \\
\hline Number-letter switching & 18 & $9.12 \pm 3.28$ & $9.47 \pm 3.39$ & .37 & 11.8 & 76.5 & 11.8 & 0.34 \\
\hline Motor Speed & 18 & $9.00 \pm 3.46$ & $8.65 \pm 3.30$ & .30 & 29.4 & 58.8 & 11.8 & -0.89 \\
\hline Letter-number switching vs letter sequencing & 18 & $9.35 \pm 2.23$ & $9.06 \pm 2.07$ & .53 & 11.8 & 82.4 & 5.9 & -0.42 \\
\hline
\end{tabular}


VERBAL FLUENCY scaled scores

\section{Letter}

Category

Category Switching

Total Correct inhibition/switching

Total set loss errors

Total repetition errors

STROOP COLOUR-WORD INTERFERENCE

scaled scores

Colour naming

Word reading

Inhibition

Inhibition/switching

Inhibition vs colour naming

$\begin{array}{lc}18 & 9.00 \pm 4.00 \\ 18 & 8.94 \pm 3.30 \\ 18 & 10.39 \pm 3.84 \\ 18 & 10.17 \pm 4.09 \\ 18 & 12.44 \pm 0.98 \\ 18 & 10.72 \pm 2.40\end{array}$

$8.17 \pm 3.31$

$$
8.72 \pm 2.70
$$

.09

$$
11.1
$$$$
9.67 \pm 1.71
$$

.69

$$
22.2
$$

88.9

0.00

$-0.65$

$\begin{array}{lll}9.67 \pm 1.71 & .47 & 27.8\end{array}$

55.6

\section{Attention}

Paced Auditory Serial Addition Test

Percent correct

$$
\begin{gathered}
9.22 \pm 2.88 \\
9.17 \pm 3.03 \\
9.33 \pm 3.41 \\
9.44 \pm 3.33 \\
10.11 \pm 2.53
\end{gathered}
$$$$
8.66 \pm 3.31
$$$$
.46
$$$$
27.8
$$$$
55.6
$$$$
-0.61
$$$$
8.50 \pm 2.87
$$$$
8.22 \pm 3.10
$$$$
.34
$$$$
22.2
$$$$
8.39 \pm 3.34
$$

.06

VTA-DBA=ventral tegmental area deep brain stimulation; D-KEFS= Delis-Kaplan Executive Function System. 
Table 3. Means and standard deviations for the measures of mood, pain, disability and quality of life before and after VTA-DBS surgery.

\begin{tabular}{|c|c|c|c|c|}
\hline Measure & $\mathbf{N}$ & Pre-Operative & Post-Operative & $\begin{array}{c}\text { corrected } \\
p=.01, \\
p=.006\end{array}$ \\
\hline \multirow[t]{2}{*}{ Beck Depression Inventory } & 18 & $23.11 \pm 11.88$ & $20.55 \pm 12.16$ & .15 \\
\hline & & $11.61 \pm 5.09$ & $10.67 \pm 5.82$ & .23 \\
\hline HADS-D & 18 & & & \\
\hline Beck Hopelessness scale & 18 & $8.61 \pm 5.43$ & $8.39 \pm 6.41$ & .62 \\
\hline Starkstein Apathy Scale & 18 & $20.83 \pm 9.19$ & $20.23 \pm 7.02$ & .70 \\
\hline HADS-A & 18 & $11.94 \pm 4.36$ & $8.00 \pm 4.51$ & $.001 *$ \\
\hline \multicolumn{5}{|l|}{$\begin{array}{l}\text { Pain } \\
\text { PAIN BEHAVIOURAL CHECK LIST }\end{array}$} \\
\hline Pain Behaviour Checklist (PBC)_total & 15 & $27.60 \pm 11.02$ & $21.78 \pm 15.09$ & .04 \\
\hline PBC Help Seeking Behaviour & 15 & $4.00 \pm 1.00$ & $2.61 \pm 1.50$ & $.006^{*}$ \\
\hline PBC Avoidance & 15 & $17.73 \pm 9.73$ & $14.94 \pm 11.39$ & .23 \\
\hline PBC Complaint & 15 & $5.87 \pm 2.23$ & $4.28 \pm 2.86$ & .08 \\
\hline McGill Pain Questionnaire & 14 & & & \\
\hline Total & 14 & $46.06 \pm 19.35$ & $50.28 \pm 15.97$ & .92 \\
\hline Sensory & 14 & $22.76 \pm 10.80$ & $26.86 \pm 8.04$ & .43 \\
\hline Affective & 14 & $8.88 \pm 4.23$ & $8.43 \pm 4.07$ & .68 \\
\hline Evaulative & 14 & $4.35 \pm 1.49$ & $4.29 \pm 1.07$ & .06 \\
\hline Miscellaneus & 14 & $10.06 \pm 5.28$ & $10.71 \pm 4.44$ & .36 \\
\hline \multicolumn{5}{|l|}{ Quality of life } \\
\hline SF-36 & 16 & & & \\
\hline SF-36 General Health & 16 & $43.85 \pm 20.77$ & $50.94 \pm 26.60$ & .21 \\
\hline SF-36 Physical functioning & 16 & $59.59 \pm 26.95$ & $62.50 \pm 27.20$ & .14 \\
\hline SF-36 Physical role functioning & 16 & $36.98 \pm 31.52$ & $37.65 \pm 39.17$ & .64 \\
\hline SF-36 Emotional role functioning & 16 & $45.98 \pm 36.23$ & $51.04 \pm 39.31$ & .68 \\
\hline SF-36 Bodily pain & 16 & $24.56 \pm 26.33$ & $39.22 \pm 34.42$ & .07 \\
\hline SF-36 Social role functioning & 16 & $28.68 \pm 29.24$ & $44.53 \pm 31.28$ & .04 \\
\hline SF-36 Energy/Fatigue & 16 & $21.76 \pm 26.39$ & $28.44 \pm 27.85$ & .15 \\
\hline SF-36 Emotional Well-being & 16 & $47.06 \pm 26.29$ & $55.44 \pm 25.84$ & .03 \\
\hline
\end{tabular}


$H A D S=$ Hospital Anxiety and Depression rating scale; $A=$ anxiety; $D=$ depression; $N=$ sample size; $P B C$ = pain behaviour checklist; SF-36 = Short Form Health Survey questionnaire; VTA-DBS =ventral tegmental area deep brain stimulation. 